\title{
Qual a relevância dos sinais e sintomas no prognóstico de pacientes com hérnia de disco lombar?
}

\author{
What is the relevance of signs and symptoms in the prognosis of \\ patients with lumbar disc herniation? \\ ¿Cuál la relevancia de los signos y síntomas en el pronóstico de \\ pacientes con hernia de disco lumbar?
}

\author{
Asdrubal Falavigna' \\ Orlando Righesso Neto ${ }^{2}$ \\ Julia Bossardi ${ }^{3}$ \\ Thiago Hoesker ${ }^{3}$ \\ Paula Caprara Gasperin ${ }^{3}$ \\ Pedro Guarise de Silva ${ }^{3}$ \\ Alisson Roberto Teles ${ }^{4}$
}

\section{RESUMO}

Introdução: a hérnia de disco lombar (HDL) é uma patologia prevalente na atualidade, que acarreta limitações físicas, psiquícas e sociais ao paciente. Os sinais e sintomas mais frequentes são lombociatalgia, distúrbios motores e sensitivos e sinal de Lasègue. Nos pacientes com HDL refrátarios ao tratamento clínico, microdiscectomia é o procedimento padrão para a melhora dos sintomas. Objetivos: estudar a relevância prognóstica dos sinais e sintomas nos pacientes com HDL refratários ao tratamento clínico. Métodos: foram pesquisados, nas principais bases de dados biomédicas, os artigos que estudaram a percentagem de melhora e o valor prognóstico dos sinais e sintomas pré-operatórios dos pacientes com HDL. Os sinais e os sintomas avaliados foram dor lombar, dor na perna, distúrbios motores

\section{ABSTRACT}

Introduction: lumbar disc herniation (LDH) is currently a prevalent pathology which leads to physical, psychological and social limitations for the patient. The most frequent signs and symptoms are lumbocyatalgia, motor and sensitive disorders and Lasègue's sign. In patients with $\mathrm{LDH}$ refractory to medical treatment, discectomy is the standard procedure to improve the symptoms. Objectives: to study the prognostic relevance of signs and symptoms in patients with LDH refractory to medical treatment. Methods: a survey was performed, in the main biomedical databases, for articles that studied the percentage of improvement and prognostic value of the preoperative signs and symptoms of patients with LDH. The signs and symptoms evaluated were lumbar pain, leg pain, motor and

\section{RESUMEN}

Introducción: la hernia de disco lumbar (HDL) es una patología prevalente en la actualidad, que implica limitaciones físicas, psíquicas y sociales al paciente. Los signos y sintomas más frecuentes son la lumbociatalgia, los disturbios motores y sensitivos y el signo de Lasègue. En los pacientes con HDL refractarios al tratamiento clínico, la microdiscectomía es el procedimiento estándar para mejoría de los síntomas. Objetivos: estudiar la relevancia del pronóstico de los signos y síntomas en los pacientes con HDL refractarios al tratamiento clínico. Métodos: se realizó una investigación, en las principales bases de datos biomédicos, de los artículos que estudiaron el porcentaje de mejoría y el valor pronóstico de los signos y síntomas preoperatorios de los pacientes con HDL. Los signos y

\footnotetext{
Departamento de Neurocirurgia e Ortopedia da Universidade de Caxias do Sul - UCS - Caxias do Sul (RS), Brasil.

1 Doutor; Professor de Neurocirurgia da Universidade de Caxias do Sul - UCS - Caxias do Sul (RS), Brasil.

2 Pós-graduanda (Doutorado) em Ciências da Saúde pela Faculdade de Ciências Médicas da Santa Casa de São Paulo - FCMSCSP - São Paulo (SP), Brasil.

${ }^{3}$ Acadêmicos do Curso de Medicina da Universidade de Caxias do Sul - UCS - Caxias do Sul (RS), Brasil.

${ }^{4}$ Residente de Neurocirurgia do Hospital São José, Santa Casa de Misericórdia - Porto Alegre (RS), Brasil. 
e sensitivos e sinal de Lasègue. Conclusão: o índice de sucesso da cirurgia dos pacientes com HDL refratários ao tratamento clínico correlacionase com a ausência de lombalgia, a presença de ciatalgia com tempo de evolução de até 6 meses, o déficit sensitivo presente de forma isolada ou associado ao déficit motor e a presença de sinal de Lasègue positivo no período pré-operatório. sensitive disorders and Lasègue's sign. Conclusion: the success rate in surgery of patients with $L D H$ refractory to medical treatment is correlated with the absence of lumbalgia, the presence of cyatalgia over up to six months, the sensitive deficit present alone or associated with motor deficit and the presence of positive Lasègue's sign during the preoperative period. sintomas evaluados fueron el dolor lumbar, el dolor en la pierna, los disturbios motores y sensitivos y el signo de Lasègue. Conclusión: el índice de éxito de la cirugía de los pacientes con HDL refractarios al tratamiento clínico se correlaciona con la ausencia de lumbalgia, la presencia de ciatalgia con tiempo de evolución de hasta seis meses, el déficit sensitivo presente de forma aislada o asociado al déficit motor y la presencia de signo de Lasègue positivo en el periodo preoperatorio.

DESCRIPTORES: Desplazamiento del disco intervertebral; Signos y síntomas; Pronóstico disco intervertebral; Sinais e sintomas; Prognóstico

\author{
KEYWORDS: Intervertebral disk \\ displacement; Signs and \\ symptoms; Prognosis
}

DESCRITORES: Deslocamento do 
TABELA 1 - Avaliação da dor nas costas como variável prognóstica

\begin{tabular}{|c|c|c|c|}
\hline Autor & Tipo de estudo & Seguimento & Prognóstico \\
\hline Katayama, 2006 & Prospectivo & 2,7 anos & Sem valor prognóstico \\
\hline Fuji, $2003^{31}$ & Prospectivo & 4,2 anos & Sem valor prognóstico \\
\hline Yorimitsu, $2001^{29}$ & Retrospectivo & 14,3 anos & Sem valor prognóstico \\
\hline Asch, $2000^{6}$ & Prospectivo & 2 anos & Sem valor prognóstico \\
\hline Junge, $1995^{23}$ & Prospectivo & 1 ano & Negativo \\
\hline Moore, $1994^{33}$ & Prospectivo & 8,6 anos & Sem valor prognóstico \\
\hline Nygaard, $1994^{42}$ & Retrospectivo & $1-3$ anos & Sem valor prognóstico \\
\hline Abramovitz, $1991^{2}$ & Prospectivo & 3 meses e 1 ano & Negativo \\
\hline Hanley, 198919 & Prospectivo & 3,2 anos & Negativo \\
\hline Dvorak, $1988^{13}$ & Retrospectivo & 10,5 anos & Negativo \\
\hline Lewis, $1987^{32}$ & Prospectivo & 5 e 10 anos & Sem valor prognóstico \\
\hline Weber, $1983^{16}$ & Prospectivo & 4 e 10 anos & Sem valor prognóstico \\
\hline
\end{tabular}

ria apresenta um valor prognóstico negativo nos pacientes com seguimento médio de 10,5 anos.

Abramovitz e Neff ${ }^{2}$ evidenciaram, em momentos diferentes de avaliação, três meses e um ano pós-operatórios, que a dor lombar é um preditor negativo de prognóstico nos pacientes submetidos à cirurgia para correção de HDL. A dor lombar preditiva de prognóstico negativo também foi observada por outros autores em diferentes tempos de seguimento ${ }^{19,23,30}$.

\section{Lombalgia sem valor prognóstico}

Fujii et al. ${ }^{31}$ não encontraram valor prognóstico relacionado à dor lombar entre pacientes com menos de 40 e mais de 65 anos, com percentual de melhora dos sintomas préoperatórios de 84,7 e $74,1 \%$, respectivamente.

Asch et al. ${ }^{6}$, utilizando a escala visual analógica (EVA) da dor, observaram que a presença da lombalgia maior ou igual à dor na perna tem valor prognóstico negativo. No entanto, quando a lombalgia é inferior à dor radicular, a variável não possui valor prognóstico no seguimento de dois anos.

Weber ${ }^{16}$ acompanhou durante dez anos os pacientes com HDL e não observou o valor prognóstico da lombalgia.

O tipo de estudo e os diferentes instrumentos utilizados para avaliação podem explicar a divergência nos resultados dos estudos referentes ao prognóstico da lombalgia nos pacientes com HDL.

\section{CIATALGIA}

A literatura demonstra comum acordo com relação à indicação do tratamento cirúrgico nos pacientes com HDL e dor radicular refratária a tratamento clínico ${ }^{16,35,36}$. O valor prognóstico da dor na perna depende não só da sua refratariedade, mas também do momento de avaliação no pós-operatório e do tempo de duração dos sintomas no período préoperatório, conforme apresentado na tabela $2^{10,13,31,34,37-42}$.

\section{Tempo de avaliação no pós-operatório}

Jonsson e Stromqvist ${ }^{10}$ observaram o mesmo percentual de melhora da dor na perna em uma avaliação de 4 e 12 meses após a cirurgia, não havendo diferença entre os tempos de seguimento e a evolução da dor.

\section{Duração dos sintomas no pré-operatório}

Fujii et al. ${ }^{31}$ verificaram que uma maior duração dos sintomas no pré-operatório possui valor prognóstico negativo na cirurgia de HDL, em especial para pacientes idosos ( 3,8 meses), com um percentual de melhora de $84,7 \%$ nos com menos de 40 anos, e de 74,1\%, nos acima de 75 anos.

Fisher et al. ${ }^{38}$ observaram que, quando o tempo de duração da dor na perna for superior a seis meses, existe um valor prognóstico negativo. $\mathrm{O}$ mesmo pôde ser observado por Nygaard et al. ${ }^{34}$ no seguimento médio de 24 meses, quando a duração dos sintomas entre 6 a 12 meses ou acima de 12 meses apresenta percentual de melhora de $32 \mathrm{e}$ de $16 \%$, respectivamente. No entanto, quando a variável foi inferior a 6 meses, o índice de melhora foi de $56 \%$, tendo um valor prognóstico positivo.

Nygaard et al. ${ }^{42}$, em seguimento de um ano, verificaram um valor prognóstico positivo para a duração do sintoma no pré-operatório quando essa variável durou menos de oito meses. O mesmo valor prognóstico foi encontrado por Hurme e Alaranta ${ }^{39}$ e por $\mathrm{Ng}$ e Sell ${ }^{41}$, porém com o tempo de duração dos sintomas no pré-operatório de 2 meses e 12 meses, respectivamente. A relação entre a duração dos sintomas dolorosos na perna e sua melhora no pós-operatório também pôde ser observada por outros autores ${ }^{13,37,40}$.

Abramovitz e Neff ${ }^{2}$ verificaram que a simples presença de dor na perna durante o pré-operatório possui valor prognóstico positivo independentemente da duração dos sintomas nesse período, sendo os pacientes avaliados após 3 e 12 meses da cirurgia.

Weber $^{16}$, em seguimentos de 4 e de 10 anos, e Moore et al..$^{33}$, em um seguimento médio de 8,6 anos, não encontraram valor prognóstico para a presença de dor na perna.

\section{Percentual de melhora da ciatalgia}

Alguns estudos relataram apenas o percentual de melhora do paciente, não sendo verificado o valor prognóstico da presença da dor na perna no pré-operatório ${ }^{4,6,19,29,32,43,44}$. 
TABELA 2 - Avaliação da dor na perna como variável prognóstica

\begin{tabular}{|c|c|c|c|c|}
\hline Autor & $\begin{array}{l}\text { Tipo de } \\
\text { estudo }\end{array}$ & $\begin{array}{c}\text { Duração da ciatalgia até } \\
\text { a cirurgia em meses } \\
\text { média (intervalo) }\end{array}$ & $\begin{array}{l}\text { Seguimento pós- } \\
\text { operatório } \\
\text { média (intervalo) }\end{array}$ & $\begin{array}{c}\text { Variável prognóstica ou } \\
\text { percentagem de melhora } \\
\text { da ciatalgia }\end{array}$ \\
\hline Katayama, $2006^{14}$ & Prospectivo & - & $\begin{array}{c}2 \text { anos e } 8 \text { meses } \\
\text { (12 meses a } 4 \text { anos) }\end{array}$ & $\begin{array}{c}\text { VAS pré-operatório: } \\
0,6 \pm 8,1 \\
\text { VAS pós-operatório: } \\
0,4 \pm 1,3\end{array}$ \\
\hline Fisher, $2004^{38}$ & Prospectivo & $\begin{array}{c}12,75 \text { meses } \\
(0,1 \text { a } 52,5 \text { meses })\end{array}$ & 6 e em 12 meses & $\begin{array}{l}\text { Prognóstico negativo se } \\
\text { duração }>6 \text { meses }\end{array}$ \\
\hline $\mathrm{Ng} \&$ Sell, $2004^{41}$ & Prospectivo & $\begin{array}{l}11 \text { meses } \\
\text { (2 a } 60 \text { meses) }\end{array}$ & 1 ano & $\begin{array}{l}\text { Prognóstico positivo se } \\
\text { duração }<12 \text { meses }\end{array}$ \\
\hline Fuji, 2003 31 & Prospectivo & $\begin{array}{c}3,0 \text { meses (grupo jovem) e } \\
3,8 \text { meses (grupo idoso) }\end{array}$ & $\begin{array}{l}21,6 \text { meses (grupo idoso) e } \\
28,5 \text { meses (grupo jovem) }\end{array}$ & $\begin{array}{l}\text { Prognóstico negativo nos } \\
\text { pacientes idosos }\end{array}$ \\
\hline Ash, $2002^{6}$ & Prospectivo & $\begin{array}{c}7,7 \pm 16,3 \text { meses } \\
(1 \text { dia } a>8 \text { anos })\end{array}$ & $\begin{array}{c}2,02 \pm 1,18 \text { anos } \\
(0,11-4,77 \text { anos })\end{array}$ & Melhora de $80 \%$ \\
\hline Yorimitsu, $2001^{29}$ & Retrospectivo & - & 14,3 anos ( 10 a 22 anos) & Melhora de $90,4 \%$ \\
\hline Nygaard, $2000^{34}$ & Retrospectivo & $\begin{array}{l}36 \text { semanas } \\
\text { (3-131 semanas) }\end{array}$ & 1 ano & $\begin{array}{l}\text { Prognóstico positivo se } \\
\text { duração }<8 \text { meses }\end{array}$ \\
\hline Takeshima, $2000^{43}$ & Prospectivo & - & $6,6 \pm 2,1$ anos & $\begin{array}{l}\text { JOA pré-operatório: } \\
0,9 \pm 0,4 \\
\text { JOA pós-operatório: } \\
2,6 \pm 0,6\end{array}$ \\
\hline Loupasis, $1999^{4}$ & Retrospectivo & - & 12,2 anos (7 a 20 anos) & Melhora de $85 \%$ \\
\hline Carragee, $1997^{37}$ & Prospectivo & $\begin{array}{c}66 \text { dias } \\
\text { (1 dia a } 2 \text { anos) }\end{array}$ & 2,6 anos & $\begin{array}{l}\text { Prognóstico positivo se } \\
\text { duração }<6 \text { meses }\end{array}$ \\
\hline Jerosch, $1996^{40}$ & Prospectivo & - & 31 anos (19 a 42 anos) & $\begin{array}{l}\text { Prognóstico positivo se } \\
\text { duração }<6 \text { meses }\end{array}$ \\
\hline Jonsson, $1995^{10}$ & Prospectivo & 13 meses & 4 a 12 meses & Melhora de $79 \%$ a $92 \%$ \\
\hline Moore, $1994^{33}$ & Prospectivo & $\begin{array}{c}\text { Divisão nos grupos de até } 3 \\
\text { meses; } 3 \text { a } 6 \text { meses e maior } \\
\text { de } 6 \text { meses }\end{array}$ & 8,6 anos (3 a 11 anos) & Sem influência \\
\hline Nygaard, $1994^{42}$ & Retrospectivo & $\begin{array}{c}9 \text { meses } \\
(0,75 \text { a } 32,75 \text { meses })\end{array}$ & 1 a 3 anos & $\begin{array}{l}\text { Prognóstico positivo se } \\
\text { duração }<6 \text { meses }\end{array}$ \\
\hline Abramovitz, $1991^{2}$ & Prospectivo & - & 3 e 12 meses & Prognóstico positivo \\
\hline Hanley, $1989^{19}$ & Prospectivo & 1,5 meses & 38 meses ( 24 a 87 meses) & Melhora de 99\% \\
\hline Dvorak, $1988^{13}$ & Retrospectivo & $\begin{array}{c}6 \text { meses } \\
(\leq 2 \text { semanas } a \geq 72 \text { meses })\end{array}$ & 10,5 anos (4 a 17 anos) & $\begin{array}{l}\text { Prognóstico positivo se } \\
\text { duração }<6 \text { meses }\end{array}$ \\
\hline Vaughan, $1988^{44}$ & Retrospectivo & 27 meses & $7,0 \pm 3,0$ anos (2 a 12 anos) & Melhora de 64\% \\
\hline Lewis, $1987^{32}$ & Prospectivo & $\begin{array}{l}16,1 \pm 3,2 \text { meses } \\
(<17 \text { meses } \geq)\end{array}$ & $\begin{array}{l}1 \text { ano } \\
10 \text { anos }\end{array}$ & $\begin{array}{l}\text { Melhora de } 96 \% \\
\text { Melhora de } 89 \%\end{array}$ \\
\hline Hurme, $1987^{39}$ & Prospectivo & 12 meses & 1 e 6 meses & $\begin{array}{l}\text { Prognóstico positivo se } \\
\text { duração }<2 \text { meses }\end{array}$ \\
\hline Weber, $1983^{16}$ & Prospectivo & 0,82 meses ( $\leq 3$ meses $\geq$ ) & 4 e 10 anos & Sem influência \\
\hline
\end{tabular}

VAS: Visual Analogue Scale; JOA: Japanese Orthopedic Association.

Asch et al. ${ }^{6}$, em um seguimento médio de 2 anos, utilizando a EVA, observaram alívio da dor na perna de $80 \%$ nos pacientes submetidos à cirurgia de HDL.

Lewis et al. ${ }^{32}$ verificaram melhora de $96 \%$ da dor na perna em um seguimento de 1 ano, e de $89 \%$ em um seguimento de 5 a 10 anos.

Yorimitsu et al. ${ }^{29}$, em seguimento médio de 14,3 anos, observaram $90,4 \%$ de melhora no pós-operatório. Loupasis et al. ${ }^{4}$, Hanley e Shapiro ${ }^{19}$ e Vaughan et al. ${ }^{44}$ en- contraram percentuais de melhora de $85 \%, 99 \%$ e $64 \%$, respectivamente.

Katayama et al. ${ }^{14}$ observaram melhora entre os valores do VAS - Visual Analogue Scale no pré-operatório $(7,9 \pm 0,6)$, e no pós-operatório $(1,2 \pm 0,4)$.

\section{DÉFICIT MOTOR E SENSITIVO}

O déficit motor e o sensitivo são sinais frequentes no exame neurológico dos pacientes com HDL ${ }^{23,26-28}$. A relação 
destes sinais com o prognóstico dos pacientes submetidos à cirurgia de HDL está apresentado na tabela $3^{2,10,16,20,23}$.

\section{Valor prognóstico}

Herron e Turner ${ }^{20}$, em estudo realizado com 114 pacientes submetidos à cirurgia de hérnia discal, verificaram que $49 \%$ deles apresentaram déficit motor e $67 \%$, déficit sensitivo no período pré-operatório. Após acompanhamento dos pacientes por 1 a 4 anos (média de 18 meses), foi observado que, quando avaliados como variáveis preditoras isoladas, o déficit motor e o déficit sensitivo não se associaram ao prognóstico do paciente. No entanto, quando ambos os déficits estiverem presentes, o prognóstico é positivo.

Junge et al. ${ }^{23}$ registraram que, no período pré-operatório, $36,6 \%$ dos pacientes apresentaram déficit motor moderado e $18,3 \%$, paralisia muscular dos membros inferiores, havendo déficit sensitivo em $75,5 \%$ deles. Após um seguimento de 12 meses, tanto a presença de déficit motor quanto a de déficit sensitivo não tinham mais valor prognóstico.

Abramovitz e $\mathrm{Neff}^{2}$ constataram que, no $3^{\circ}$ e no $12^{\circ}$ mês de pós-operatório, a presença de déficit sensitivo aumenta a probabilidade de um desfecho positivo para a cirurgia de hérnia discal. Não há trabalhos que definam o déficit sensitivo como variável negativa para o prognóstico dos pacientes com HDL.

Dvorak et al. ${ }^{13}$ relataram que pacientes com déficit sensório-motor e reflexos tendinosos ausentes mostram melhores resultados a longo prazo.

\section{Índice de melhora motora comparando o tratamento cirúrgico e o clínico}

Yorimitsu et al. ${ }^{29}$ acompanharam 131 pacientes submetidos à discectomia por HDL por um período de 10 a 22 anos (média de 14,3 anos). No período pré-operatório, $76,2 \%$ dos pacientes apresentaram déficit motor e $81 \%$, déficit sensitivo, havendo uma melhora com a cirurgia de 14,3 e $31,7 \%$, respectivamente.

Bloch-Michel et al ${ }^{45}$ relataram melhora da paresia em $64 \%$ dos pacientes tratados com a cirurgia, e apenas em
$56 \%$ dos submetidos a tratamento não-cirúrgico. Este dado também foi encontrado por Weigert ${ }^{46}$, que evidenciou uma melhora mais acentuada da paresia nos pacientes submetidos à cirurgia se comparados com os tratados clinicamente (77 versus $53 \%$ ).

De Seze et al. ${ }^{47}$ encontraram resultados que se contrapõem aos anteriormente citados. Demonstrou-se que $63 \%$ dos pacientes tratados de forma não-cirúrgica apresentaram melhora na paresia, enquanto com a cirurgia o valor foi de $54 \%$. O estudo de Weber ${ }^{16}$ demonstrou uma taxa de recuperação similar, em torno de $70 \%$, independentemente de o tratamento ser cirúrgico ou clínico.

\section{Intensidade e duração da paresia e recuperação}

Segundo Eysel et al. ${ }^{48}$, o grau de intensidade da paresia pode servir como um bom indicador prognóstico. Nesse estudo, mais de $70 \%$ dos casos de paresia grau 3 e 4 retrocederam em 6 meses, enquanto a taxa de recuperação nos pacientes com paresia grau 2 foi de $40 \%$ e não houve registro de recuperação nos casos de paresia total.

Woertgen et al. ${ }^{28} \mathrm{e}$ Weigert ${ }^{46}$ concluíram que a duração da paresia no período pré-operatório tem relação com mau prognóstico para sua recuperação. Entretanto, outros trabalhos demonstraram não haver relação entre a duração da paresia no pré-operatório e a recuperação do déficit motor após a cirurgia ${ }^{48,49}$. Kobayashi et al. ${ }^{50}$, no estudo experimental com animais in vivo, demonstraram que a compressão radicular causa uma reação axonal que, por sua vez, ocasiona uma degeneração retrógrada do neurônio motor no corno anterior da medula. Portanto, pacientes portadores de compressão radicular de longa evolução devem estar cientes de que os sintomas podem não regredir de imediato após a cirurgia.

Postachini $^{36}$, em seu estudo prospectivo com 116 pacientes portadores de déficit motor secundário à hérnia de disco lombar e submetidos à microdiscectomia, concluiu que o grau de recuperação da função motora era inversamente relacionado à severidade e duração do déficit muscular pré-operatório, sendo esta menor que dois meses.

\section{TABELA 3 - Avaliação do déficit sensitivo como variável prognóstica}

\begin{tabular}{lccc}
\hline Autor & Tipo de estudo & Tempo de observação & Prognóstico \\
\hline Yorimitsu, 200121 & Retrospectivo & 10 a 22 anos (14,3 anos) & Positivo \\
Junge, $1995^{23}$ & Prospectivo & 12 meses & Sem valor prognóstico \\
Abramovitz, 19912 & Prospectivo & 12 meses & Positivo \\
Dvorak, 1988'3 & Retrospectivo & 4 a 17 anos & Positivo \\
Herron, $1985^{20}$ & Prospectivo & 1 a 4 anos (18 meses) & Sem valor prognóstico \\
\hline
\end{tabular}

TABELA 4 - Avaliação do sinal de Lasègue como variável prognóstica

\begin{tabular}{lccc}
\hline Autor & Tipo de estudo & Tempo de observação & Variável prognóstica \\
\hline Junge, $1995^{23}$ & Prospectivo & 1 ano & Sem valor prognóstico \\
Abramovitz, 19912 & Prospectivo & 3 meses & Positivo \\
Dvorak, 1988 193 & Retrospectivo & 4 a 17 anos & Positivo \\
Herron, $1985^{20}$ & Prospectivo & 1 ano & Positivo \\
\hline
\end{tabular}




\section{SINAL DE LASÈGUE}

O sinal de Lasègue é um dos sinais clínicos mais comumente encontrados em pacientes com $\mathrm{HDL}^{26}$. O teste de Lasègue é considerado um importante fator preditor de necessidade da cirurgia e de prognóstico nos pacientes com HDL, sendo estes dados descritos na tabela $4^{2,13,20,23}$.

Abramovitz et al. ${ }^{13}$ e Herron e Turner ${ }^{20}$ constataram que pacientes com sinal de Lasègue positivo no pré-operatório apresentam melhor prognóstico cirúrgico no tempo de observação entre 3 meses a 17 anos. Lewis et al. ${ }^{32}$ observaram que os pacientes com sinal de Lasègue positivo no pré-operatório apresentaram 63 e 59\% de melhora na dor nas costas no período de observação de 1 e 10 anos, respectivamente, e, em relação à dor na perna, a melhora foi observada em 79 e $61 \%$. Entretanto, Junge et al. ${ }^{23}$, em estudo prospectivo com 381 pacientes avaliados no primeiro ano pós-operatório, mostraram que a presença do sinal de Lasègue positivo no pré-operatório não altera o prognóstico dos pacientes com HDL.

\section{CONCLUSÃO}

A diversidade metodológica dos estudos prospectivos e retrospectivos, o número de pacientes, o tempo de observação, o uso de diferentes técnicas de discectomia e os diversos instrumentos de avaliação dificultam uma análise comparativa dos resultados. Há uma tendência na literatura de que o paciente ideal para o tratamento cirúrgico de HDL refratária ao tratamento clínico é aquele que apresenta, no período pré-operatório, a ciatalgia como sintoma predominante à lombalgia de evolução de até seis meses, o déficit sensitivo presente de forma isolada ou associado ao déficit motor e a presença do sinal de Lasègue.

\section{REFERÊNCIAS}

1. Rebain R, Baxter GD, McDonough S. A systematic review of the passive straight leg raising test as a diagnostic aid for low back pain (1989 to 2000). Spine (Phila Pa 1976). 2002;27(17):E388-95.

2. Abramovitz JN, Neff SR. Lumbar disc surgery: results of the Prospective Lumbar Discectomy Study of the Joint Section on Disorders of the Spine and Peripheral Nerves of the American Association of Neurological Surgeons and the Congress of Neurological Surgeons. Neurosurgery. 1991;29(2):301-7; discussion 7-8.

3. Davis RA. A long-term outcome analysis of 984 surgically treated herniated lumbar discs. J Neurosurg. 1994;80(3):415-21.

4. Loupasis GA, Stamos K, Katonis PG, Sapkas G, Korres DS, Hartofilakidis G. Seven- to 20-year outcome of lumbar discectomy. Spine (Phila Pa 1976). 1999;24(22):2313-7.

5. Deville WL, van der Windt DA, Dzaferagic A, Bezemer PD, Bouter LM. The test of Lasegue: systematic review of the accuracy in diagnosing herniated discs. Spine (Phila Pa 1976). 2000;25(9):1140-7.

6. Asch HL, Lewis PJ, Moreland DB, Egnatchik JG, Yu YJ, Clabeaux DE, et al. Prospective multiple outcomes study of outpatient lumbar microdiscectomy: should 75 to $80 \%$ success rates be the norm? J Neurosurg. 2002;96(1 Suppl):34-44.
7. Ebeling U, Reichenberg W, Reulen HJ. Results of microsurgical lumbar discectomy. Review on 485 patients. Acta Neurochir (Wien). 1986;81(12):45-52.

8. Ebeling U, Kalbarcyk H, Reulen HJ. Microsurgical reoperation following lumbar disc surgery. Timing, surgical findings, and outcome in 92 patients. J Neurosurg. 1989;70(3):397-404.

9. Findlay GF, Hall BI, Musa BS, Oliveira MD, Fear SC. A 10-year follow-up of the outcome of lumbar microdiscectomy. Spine (Phila Pa 1976). 1998;23(10):1168-71.

10.Jonsson B, Stromqvist B. Influence of age on symptoms and signs in lumbar disc herniation. Eur Spine J. 1995;4(4):202-5.

11. McCulloch JA. Focus issue on lumbar disc herniation: macro- and microdiscectomy. Spine (Phila Pa 1976). 1996;21(24 Suppl):45S-56S.

12.Wenger M, Mariani L, Kalbarczyk A, Groger U. Long-term outcome of 104 patients after lumbar sequestrectomy according to Williams. Neurosurgery. 2001;49(2):329-34; discussion 34-5.

13.Dvorak J, Gauchat MH, Valach L. The outcome of surgery for lumbar disc herniation. I. A 4-17 years' follow-up with emphasis on somatic aspects. Spine (Phila Pa 1976). 1988;13(12):1418-22.
14.Katayama Y, Matsuyama Y, Yoshihara H, Sakai Y, Nakamura H, Nakashima $\mathrm{S}$, et al. Comparison of surgical outcomes between macro discectomy and micro discectomy for lumbar disc herniation: a prospective randomized study with surgery performed by the same spine surgeon. J Spinal Disord Tech. 2006;19(5):344-7.

15.Righesso O, Falavigna A, Avanzi O. Comparison of open discectomy with microendoscopic discectomy in lumbar disc herniations: results of a randomized controlled trial. Neurosurgery. 2007;61(3):545-9; discussion 9.

16. Weber H. Lumbar disc herniation. A controlled, prospective study with ten years of observation. Spine (Phila Pa 1976). 1983;8(2):131-40.

17.Smyth H, Gallagher J, McManus F. Surgery in lumbar disc protrusion-a long-term follow-up. Ir Med J. 1983;76(1):25-6.

18. Weinstein JN, Lurie JD, Tosteson TD, Tosteson AN, Blood EA, Abdu WA, et al. Surgical versus nonoperative treatment for lumbar disc herniation: four-year results for the Spine Patient Outcomes Research Trial (SPORT). Spine (Phila Pa 1976). 2008;33(25):2789-800.

19.Hanley EN Jr., Shapiro DE. The development of low-back pain after excision of a lumbar disc. J Bone Joint Surg Am. 1989;71(5):719-21. 
20.Herron LD, Turner J. Patient selection for lumbar laminectomy and discectomy with a revised objective rating system. Clin Orthop Relat Res. 1985;(199):145-52.

21.Andersson GB, Brown MD, Dvorak J, Herzog RJ, Kambin P, Malter A, et al. Consensus summary of the diagnosis and treatment of lumbar disc herniation. Spine (Phila $\mathrm{Pa}$ 1976). 1996;21(24 Suppl):75S-8S.

22.Deyo RA, Rainville J, Kent DL. What can the history and physical examination tell us about low back pain? JAMA. 1992;268(6):7605. Comment in: JAMA. 1993 Jan 20;269(3):355-6; JAMA. 1993 Jan 20;269(3):355; author reply 356; JAMA. 1993 Jan 20;269(3):355; author reply 356; JAMA. 1993 Jan 20;269(3):354-5; author reply 356; JAMA. 1993 Jan 20;269(3):354; author reply 356 .

23.Junge A, Dvorak J, Ahrens S. Predictors of bad and good outcomes of lumbar disc surgery. A prospective clinical study with recommendations for screening to avoid bad outcomes. Spine (Phila Pa 1976). 1995;20(4):460-8.

24.Mannion AF, Elfering A, Staerkle R, Junge A, Grob D, Dvorak J, et al. Predictors of multidimensional outcome after spinal surgery. Eur Spine J. 2007;16(6):777-86.

25.Jensen MC, Brant-Zawadzki MN, Obuchowski N, Modic MT, Malkasian D, Ross JS. Magnetic resonance imaging of the lumbar spine in people without back pain. N Engl J Med. 1994;331(2):69-73.

26.Kortelainen P, Puranen J, Koivisto E, Lahde S. Symptoms and signs of sciatica and their relation to the localization of the lumbar disc herniation. Spine (Phila Pa 1976). 1985;10(1):88-92.

27.Postacchini F, Giannicola G, Cinotti G. Recovery of motor deficits after microdiscectomy for lumbar disc herniation. J Bone Joint Surg Br. 2002;84(7):1040-5.

28. Woertgen C, Rothoerl RD, Breme K, Altmeppen J, Holzschuh M, Brawanski A. Variability of outcome after lumbar disc surgery. Spine (Phila Pa 1976). 1999;24(8):807-11.

29. Yorimitsu E, Chiba K, Toyama Y, Hirabayashi K. Long-term outcomes of standard discectomy for lumbar disc herniation: a follow-up study of more than 10 years. Spine (Phila Pa 1976). 2001;26(6):652-7.
30.LaMont RL, Morawa LG, Pederson HE. Comparison of disk excision and combined disk excision and spinal fusion for lumbar disk ruptures. Clin Orthop Relat Res. 1976;(121):212-6.

31.Fujii K, Henmi T, Kanematsu Y, Mishiro T, Sakai T. Surgical treatment of lumbar disc herniation in elderly patients. J Bone Joint Surg Br. 2003;85(8):1146-50.

32.Lewis PJ, Weir BK, Broad RW, Grace MG. Long-term prospective study of lumbosacral discectomy. J Neurosurg. 1987;67(1):49-53.

33.Moore AJ, Chilton JD, Uttley D. Long-term results of microlumbar discectomy. Br J Neurosurg. 1994;8(3):319-26.

34.Nygaard OP, Romner B, Trumpy JH. Duration of symptoms as a predictor of outcome after lumbar disc surgery. Acta Neurochir (Wien). 1994;128(14):53-6.

35.Atlas SJ, Keller RB, Wu YA, Deyo RA, Singer DE. Long-term outcomes of surgical and nonsurgical management of sciatica secondary to a lumbar disc herniation: 10 year results from the maine lumbar spine study. Spine (Phila Pa 1976). 2005;30(8):927-35.

36.Postacchini F. Results of surgery compared with conservative management for lumbar disc herniations. Spine (Phila Pa 1976). 1996;21(11):1383-7.

37.Carragee EJ, Kim DH. A prospective analysis of magnetic resonance imaging findings in patients with sciatica and lumbar disc herniation. Correlation of outcomes with disc fragment and canal morphology. Spine (Phila Pa 1976). 1997;22(14):1650-60.

38.Fisher C, Noonan V, Bishop P, Boyd M, Fairholm D, Wing P, et al. Outcome evaluation of the operative management of lumbar disc herniation causing sciatica. J Neurosurg. 2004;100(4 Suppl Spine):317-24.

39.Hurme M, Alaranta H. Factors predicting the result of surgery for lumbar intervertebral disc herniation. Spine (Phila Pa 1976). 1987;12(9):933-8.

40.Jerosch J, Castro WH. [Long-term results in revision surgery following lumbar disk nucleotomy]. Z Orthop Ihre Grenzgeb. 1996;134(1):89-96.

41.Ng LC, Sell P. Predictive value of the duration of sciatica for lumbar discectomy. A prospective cohort study. J Bone Joint Surg Br. 2004;86(4):546-9.
42. Nygaard OP, Kloster R, Solberg T. Duration of leg pain as a predictor of outcome after surgery for lumbar disc herniation: a prospective cohort study with 1-year follow up. J Neurosurg. 2000;92(2 Suppl):131-4.

43. Takeshima T, Kambara K, Miyata S, Ueda Y, Tamai S. Clinical and radiographic evaluation of disc excision for lumbar disc herniation with and without posterolateral fusion. Spine (Phila $\mathrm{Pa}$ 1976). 2000;25(4):450-6.

44. Vaughan PA, Malcolm BW, Maistrelli GL. Results of L4-L5 disc excision alone versus disc excision and fusion. Spine (Phila Pa 1976). 1988;13(6):690-5.

45.Bloch-Michel H, Cauchoix J, Benoist M. [Apropos of 60 cases of paralytic sciatica]. Sem Hop. 1967;43(43): 2640-6.

46. Weigert M. [The regression of neurological symptoms following intervertebral disk surgery]. Z Orthop Ihre Grenzgeb. 1967;103(3):294-8.

47.De Seze S, Guillaume J, DesprogesGotteron R, Jurmand SH. [Paralytic sciatica; clinical pathogenic \& therapeutic study based on 100 cases]. Sem Hop. 1957;33(28):1773-96.

48. Eysel P, Rompe JD, Hopf C. Prognostic criteria of discogenic paresis. Eur Spine J. 1994;3(4):214-8.

49.Knutsson B. How often do the neurological signs disappear after the operation of a herniated disc? Acta Orthop Scand. 1962;32:352-6.

50.Kobayashi S, Uchida K, Yayama T, Takeno K, Miyazaki T, Shimada $\mathrm{S}$, et al. Motor neuron involvement in experimental lumbar nerve root compression: a light and electron microscopic study. Spine (Phila $\mathrm{Pa}$ 1976). 2007;32(6):627-34.

Correspondência
Asdrubal Falavigna
Faculdade de Medicina da Universidade
de Caxias do Sul
Rua General Arcy da Rocha Nóbrega,
401/602
CEP: 95050-290 - Caxias do Sul (RS),
Brasil
Fone/Fax: (54) 3222-0684
E-mail: asdrubalmd@gmail.com

